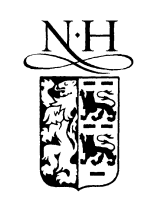

ELSEVIER

\title{
Analysis of gray level corner detection ${ }^{1}$
}

\author{
Zhiqiang Zheng, Han Wang *, Eam Khwang Teoh
}

School of Electrical and Electronic Engineering, Nanyang Technological University, Singapore 639798, Singapore

Received 29 May 1998; received in revised form 9 October 1998

\begin{abstract}
In this paper the analysis of gray level corner detection has been carried out. Performances of various cornerness measures are discussed with respect to four performances of robustness: detection, localization, stability and complexity. We have analyzed the interior differential features of the image surface of these cornerness measures. This paper presents a new approach called gradient-direction corner detector for the corner detection which is developed from the popular Plessey corner detection. The gradient-direction corner detector is based on the measure of the gradient module of the image gradient direction and the constraints of the false corner response suppression. (c) 1999 Elsevier Science B.V. All rights reserved.
\end{abstract}

Keywords: Feature extraction; Gray level corner; Corner detection; Differential geometry

\section{Introduction}

In this paper, the term gray level corner denotes the gray-level transitions in a sufficiently large area around the prominent point of the gray level corner. This gray level corner point is usually defined as the point of maximal planar curvature in the line of the steepest gray-level slope (Rohr, 1992; Guiducci, 1988). Corner detection can simply mean to detect and localize this prominent point or can, in addition, include the determination of inherent attributes. In the scale space, each line pattern describing the corner location either persists, terminates or merges with a neighboring pattern. Moreover, new additional line patterns will not be introduced by smoothing (Rattarangsi and Chin, 1992).

There are mainly two categories of gray level corner detections, namely, template based corner detection and geometry based corner detection. Four performances of robustness must be addressed by all of the corner detections to compare their advantages and shortcomings.

- Detection. The corner detection should detect even the very subtle corners, with ignoring noise effects.

- Localization. The corners should be detected as close as possible to their true locations.

- Stability. The detected position of a corner should not move when multiple images are acquired of the same scene.

\footnotetext{
${ }^{*}$ Corresponding author. E-mail: hw@ntu.edu.sg

${ }^{1}$ Electronic Annexes available. See http://www.locate.patrec.
} 
- Complexity. The reduced algorithm complexity contribute to more automation process and faster implementation.

We have defined several cornerness measure in the gray level images and implement corner detectors based on these measures.

\section{Template based corner detection}

Template based corner detection involves determining the similarity, or correlation, between a given template size $n \times n$ and all sub-windows of size $n \times n$ in a given image (Singh and Shneier, 1990).

We assume that the template is square, $n=2 k+1$. Let $p_{i, j}$ and $q_{i, j}$ represent the $(i, j)$ element of the template $P$ and the image $Q$. In the template we define the mean $\alpha$ and the variance $\sigma$ and in a window $n \times n$ centered at the $(i, j)$ pixel of the image the mean $\beta$ and the variance $\sigma_{i, j}$. Then a generalized correlation between the image and the template at the $(i, j)$ pixel can be defined as

$$
\sigma_{i, j}=\frac{\frac{1}{n} \sum_{l=-k}^{k} \sum_{m=-k}^{k}\left(p_{l, m}-\alpha\right)\left(q_{i+l, j+m}-\beta\right)}{\sqrt{\sigma(p) \sigma(q)_{i, j}}} .
$$

This algorithm has been implemented as follows: first we generate eight masks by rotating the masks in steps of $\pi / 4$, then obtain the cornerness measure at a pixel by computing the appropriate correlation measure with each of the eight masks and finally select the maximum value as the detected corners.

Unfortunately it is impossible to design the templates which can cover all orientations and corner angles. Thus, the error is inevitable because of the complicated structure of the corners.

\section{Geometry based corner detection}

Geometry based corner detection relies on measuring the differential geometry features of corner points. The methods can be divided into three ways: edge-related corner detection, topology corner detection and auto-correlation corner detection.

\subsection{Edge-related corner detection}

Edge-related corner detection considers the corner point as the junction of two or more edge lines. So that the corner point becomes the additional feature of edge point.

The approaches can be done by two ways.

One approach treats it as the two stage processes of edge detection and edge grouping followed by digital curve representation. The corner points can be found as follows:

- Extract edges as a chain code, then search for points having maximal curvature estimated by using pixel coordinates of the chain code (Cooper et al., 1993), the partial derivatives of the image $I(x, y)$ with respect to $x$ and $y$ (Ponce and Brady, 1985).

- Extract edges as a chain code, perform a polygonal approximation on the chains and then search for the line segment intersections (Hsin-Teng and $\mathrm{Hu}, 1996$ ).

- Use Mathematical Morphology to extract the convex and concave points from the edge points (Alison Noble, 1989; Lin et al., 1998). 
The other approach makes the use of the differential geometry operators from the standpoint of edge detector performance. The cornerness measures are expressed as a function of the edge-strength (which is a function of the intensity gradient) and the gradient of edge-direction.

Kitchen and Rosenfeld (1982) proposed a cornerness measure based on the change of gradient direction along an edge contour multiplied by the local gradient magnitude. Denote $I_{x}=\partial I / \partial x$ and $I_{y}=\partial I / \partial y$ as the first-order gradient multiple images and $I_{x x}=\partial^{2} I / \partial x^{2}, I_{x y}=\partial^{2} I / \partial x y$ and $I_{y y}=\partial^{2} I / \partial y^{2}$ as the second-order gradient multiple images, the gradient direction of $\theta(x, y)$ at any point $(x, y)$ is $\tan (\theta)=I_{y} / I_{x}$. The cornerness measure is defined as follows:

$$
\Delta=\left(-I_{y}, I_{x}\right)\left(\theta_{x}, \theta_{y}\right)=\frac{I_{x x} I_{y}^{2}-2 I_{x y} I_{x} I_{y}+I_{y y} I_{x}^{2}}{I_{x}^{2}+I_{y}^{2}} .
$$

The local maximum of this measure isolated corners using a non-maximum suppression applied on the gradient magnitude before its multiplication with the curvature.

Some properties of the Kitchen and Rosenfeld corner detector:

- Only the negative minimum of the cornerness measure exists.

- The corner detector is sensitive to the noise effects. Even worse, it falsely detect the edge points on which one principal curvature is large and the other principal curvature's magnitude fluctuates significantly with noise.

- Due to the rounding effect, the detected corner points are not well localized near the corners, especially when the edge near the corner is blurred.

- The corner detector does not detect consistently the same corner points from one image to the next and thus it is instable.

- The corner detector algorithm only needs to decide the thresholding value.

- The corner detector algorithm requires $M^{2}\left(5 N^{2}+10\right)$ operations where the image is $M$ by $M$ and the neighborhood of the gradient operators is $N^{2}$.

Wang and Brady (1995) proposed a corner detection algorithm based on the measurement of surface curvature. Let the image be $I(x, y)$, the gradient vector $\nabla I=\left(I_{x}, I_{y}\right)$, the edge normal $n=\nabla I /|\nabla I|$ and the edge tangential $t$, the Laplacian image $\nabla^{2} I=I_{x x}+I_{y y}$, the total curvature $\kappa$ of the image surface is defined to be

$$
\kappa \approx \frac{\partial^{2} I}{\partial t^{2}} /|\nabla I|, \quad \text { where } \nabla^{2} I \gg 1 .
$$

The equation above means that the total curvature proportional to the second derivative along the edge tangential $t$ and is inversely proportional to the edge strength. Using the false response suppression, the cornerness measure becomes

$$
\Delta=\left(\frac{\partial^{2} I}{\partial t^{2}}\right)^{2}-S|\nabla I|^{2} .
$$

Suppose that the corner points are also edge points, the cornerness measure simply is

$$
\Delta=\left(\nabla^{2} I\right)^{2}-S|\nabla I|^{2} .
$$

The Wang and Brady corner detector simplifies the cornerness measure thus it can be used in real-time corner detection for motion estimation. The thresholding value and the parameter for the false corner response suppression need to be decided and the algorithm only requires $M^{2}\left(4 N^{2}+2\right)$ operations where the image is $M$ by $M$ and the neighborhood of the gradient operators is $N^{2}$. The performance of Wang corner detection can well satisfy the requirement of the real-time algorithm. 


\subsection{Topology corner detection}

Topology corner detection considers corner point as the interior geometric feature point in the image surface. It work directly on a gray-level image. These corner detectors are based on the measurement of the topological feature of differential geometry of corner in the image surface.

Beaudet (1978) proposed a rotationally invariant measure which derived from the Hessian Matrix $H$, second-order Taylor expression of intensity surface

$$
\Delta=\operatorname{det}(H)=\left|\begin{array}{cc}
I_{x x} & I_{x y} \\
I_{x y} & I_{y y}
\end{array}\right| .
$$

The corner detection is based on the thresholding of the maximum of this cornerness measure.

Some properties of the Beaudet corner detector:

- The zero of the derivative of the measure of cornerness include two kinds of points: positive maximums and negative minimums.

- There are falsely detected negative minimums in the straight oblique line, especially when the edge is strong and noise-effected.

- The positive maximum of Beaudet corner detector can well detect the corner point, but they are some sensitive to noise.

- The positive maximum of Beaudet corner detector does not correctly locate the corner point, they are not stable in the scale space.

- The corner detector provides a local maximum in all directions and hence it is quite stable.

- The corner detector algorithm only needs to decide the thresholding value.

- The corner detector algorithm requires $M^{2}\left(3 N^{2}+2\right)$ operations where the image is $M$ by $M$ and the neighborhood of the gradient operators is $N^{2}$.

Deriche and Giraudon (1993) developed the algorithm above by making use of two important properties of the corner:

- The well-known property that the Laplacian image is zero at the exact position of the corners.

- In the scale space the positive maximum of Beaudet detector moves along the bisector line of the corner.

The implementation of the Deriche corner detection is summarized here:

1. A Laplacian image is calculated.

2. Beaudet detections at several scales $(\sigma=1, \sigma=1.5$ and $\sigma=2$ are used) are calculated and a positivemaximum detection in all directions is performed. Around each detected positive maximum in the image corresponding to the first scale, look in the next scaled image for the position of the corresponding maximum, then link to the position of the last maximum in scaled image.

3. Once these maximums are detected, look for the exact position of the corner as the point on the line segment joining the corner position in first scaled image and the corner position in last scaled image where a zero-crossing occurs in the Laplacian image.

Some properties of the Deriche corner detector:

- It is not clear whether sensitivity to noise and reliability of detection is improved.

- Almost all features can be well detected and localized in each image.

- The corner detector is unstable.

- The corner detector algorithm needs to decide the Gaussian scales, the thresholding values for each scaled images and the window size to link the corresponding maximums in the scaled image - so complicated.

- The corner detector algorithm requires $M^{2}\left((3 p+2) N^{2}+(p+1) n^{2}+p m^{2}\right)$ operations where the image is $M$ by $M$, the neighborhood of the gradient operators is $N^{2}$, the number of the Gaussian convolutions is $p$ and the Gaussian window is $n^{2}$ and the link window is $m^{2}-$ so time-consuming. 


\subsection{Auto-correlation corner detection}

Moravec (1977) proposed a corner detector which functions by considering a local window in the image, and determining the average changes of intensity which result from shifting the window by a small amount in various directions. A corner can be detected by finding when the minimum change produced by any of the shift is larger than a thresholding value. Denoting the image intensities by $I$, the change $E$ produced by a shift $(x, y)$ is given by

$$
E_{x y}=\sum_{u, v} W_{u, v}[I(u+x, v+y)-I(u, v)]^{2},
$$

where $W$ specifies the current image window. Thus, Moravec corner detection is simply this: look for local maximum in $\min \left\{E_{x y}\right\}$, above a thresholding value.

Harris et al. (see (Alison Noble, 1988)) have performed an analytic expansion about the shift origin as follows:

$$
E_{x y}=\sum_{u, v} W_{u, v}\left[x I_{x}+y I_{y}+\mathrm{O}\left(x^{2}, y^{2}\right)\right]^{2} \approx(x, y) M(x, y)^{\mathrm{T}}
$$

where

$$
M=\left(\begin{array}{cc}
\hat{I}_{x}^{2} & \widehat{I}_{x} I_{y} \\
\widehat{I_{x} I_{y}} & \hat{I}_{y}^{2}
\end{array}\right), \quad \hat{I}_{x}^{2}=I_{x}^{2} \otimes W, \quad \hat{I}_{y}^{2}=I_{y}^{2} \otimes W, \quad \widehat{I}_{x} I_{y}=\left(I_{x} I_{y}\right) \otimes W
$$

and $\mathrm{O}\left(x^{2}, y^{2}\right)$ represents higher order properties. $W$ is defined as the Gaussian function for a smooth circular window. This cornerness measure is also referred to as the Plessey Corner Detector.

Let $\alpha$ and $\beta$ be the eigenvalues of $M, \alpha$ and $\beta$ will be proportional to the principal curvatures, and form a rotational invariant description of $M$. When and only when both curvatures are larger than some thresholding values which means the shifts in any direction will leads to significantly change in $E$, a corner is indicated in the image.

The cornerness measure is defined as

$$
\begin{gathered}
\Delta=\left(\hat{I}_{x}^{2} \hat{I}_{y}^{2}-\widehat{I_{x} I_{y}^{2}}\right)-K\left(\hat{I}_{x}^{2}+\hat{I}_{y}^{2}\right)^{2}=(1-2 K) \alpha \beta-K\left(\alpha^{2}+\beta^{2}\right), \\
\text { where } K=\frac{t}{(1+t)^{2}} \quad \text { and } \quad \frac{1}{t}<\frac{\alpha}{\beta}<t
\end{gathered}
$$

$K$ is a constant variable which varies with different differentiation masks and different Gaussian convolution.

Some properties of the Plessey corner detector:

- The corner operator is the best behaved with respect to detection, however it has a poor localization performance.

- The corner detector is instable because of the application of constant-variable false corner response suppression.

- The corner detector algorithm needs to decide the thresholding value, the Gaussian scale value and the variable $K_{\text {Plessey }}$ for the false corner response suppression.

- The corner detector algorithm requires $M^{2}\left(2 N^{2}+3 n^{2}+7\right)$ operations where the image is $M$ by $M$, the neighborhood of the gradient operators is $N^{2}$ and the Gaussian window is $n^{2}$. 


\section{The new corner detector}

First we analyze the Plessey cornerness measure $\Delta(x, y)=C_{1}(x, y)-K C_{2}(x, y)$. Let $g(x, y)$ to represent the Gaussian weighted coefficient at the location $(x, y)$ within the image window, that is,

$$
g(x, y)=\frac{1}{2 \pi \sigma^{2}} \mathrm{e}^{\left(x^{2}+y^{2}\right) / 2 \sigma^{2}} .
$$

Then the Plessey cornerness measure can be expressed as follows:

$$
\begin{aligned}
& C_{1}(x, y)= \hat{I}_{x}^{2} \hat{I}_{y}^{2}-\left(\widehat{I_{x} I_{y}}\right)^{2} \\
&= \sum_{x_{1}, y_{1}} g\left(x_{1}, y_{1}\right) I_{x}^{2}\left(x-x_{1}, y-y_{1}\right) \sum_{x_{2}, y_{2}} g\left(x_{2}, y_{2}\right) I_{y}^{2}\left(x-x_{2}, y-y_{2}\right) \\
&-\left[\sum_{x_{3}, y_{3}} g\left(x_{3}, y_{3}\right) I_{x}\left(x-x_{3}, y-y_{3}\right) I_{y}\left(x-x_{3}, y-y_{3}\right)\right]^{2} \\
&= \sum_{x_{1}, y_{1} x_{2}, y_{2}} g\left(x_{1}, y_{1}\right) g\left(x_{2}, y_{2}\right)\left[I_{x}^{2}\left(x-x_{1}, y-y_{1}\right) I_{y}^{2}\left(x-x_{2}, y-y_{2}\right)\right. \\
&\left.-I_{x}\left(x-x_{1}, y-y_{1}\right) I_{y}\left(x-x_{1}, y-y_{1}\right) I_{x}\left(x-x_{2}, y-y_{2}\right) I_{y}\left(x-x_{2}, y-y_{2}\right)\right] \\
&= \frac{1}{2} \sum_{x_{1}, x_{2}, y_{1}, y_{2}} g\left(x_{1}, y_{1}\right) g\left(x_{2}, y_{2}\right)\left[I_{x}\left(x-x_{1}, y-y_{1}\right) I_{y}\left(x-x_{2}, y-y_{2}\right)\right. \\
&\left.\quad-I_{x}\left(x-x_{2}, y-y_{2}\right) I_{y}\left(x-x_{1}, y-y_{1}\right)\right]^{2} \\
&= \frac{1}{2} \sum_{x_{1}, x_{2}, y_{1}, y_{2}} g\left(x_{1}, y_{1}\right) g\left(x_{2}, y_{2}\right)\left[( I _ { x } ( x - x _ { 1 } , y - y _ { 1 } ) , I _ { y } ( x - x _ { 1 } , y - y _ { 1 } ) ) \left(-I_{y}\left(x-x_{2}, y-y_{2}\right),\right.\right. \\
&\left.\left.I_{x}\left(x-x_{2}, y-y_{2}\right)\right)\right]^{2} .
\end{aligned}
$$

Note the following equalities:

$$
\begin{aligned}
& I_{x}\left(x+x_{0}, y+y_{0}\right) \approx I_{x}(x, y)+x_{0} I_{x x}(x, y)+y_{0} I_{x y}(x, y), \\
& I_{y}\left(x+x_{0}, y+y_{0}\right) \approx I_{y}(x, y)+x_{0} I_{x y}(x, y)+y_{0} I_{y y}(x, y) . \\
& C_{1}(x, y) \approx \frac{1}{2} \sum_{x_{1}, x_{2}, y_{1}, y_{2}} g\left(x_{1}, y_{1}\right) g\left(x_{2}, y_{2}\right)\left[\left(I_{x}(x, y)-x_{1} I_{x x}(x, y)-y_{1} I_{x y}(x, y),\right.\right. \\
&\left.I_{y}(x, y)-x_{1} I_{x y}(x, y)-y_{1} I_{y y}(x, y)\right)\left(I_{x}(x, y)-x_{2} I_{x x}(x, y)-y_{2} I_{x y}(x, y),\right. \\
&\left.\left.I_{y}(x, y)-x_{2} I_{x y}(x, y)-y_{2} I_{y y}(x, y)\right)\right]^{2} \\
&= \frac{1}{2} \sum_{x_{1}, x_{2}, y_{1}, y_{2}} g\left(x_{1}, y_{1}\right) g\left(x_{2}, y_{2}\right)\left[\left(x_{1}-x_{2}\right)\left(I_{x y} I_{x}(x, y)-I_{x x} I_{y}(x, y)\right)\right. \\
&\left.+\left(y_{1}-y_{2}\right)\left(I_{y y} I_{x}(x, y)-I_{x y} I_{y}(x, y)\right)-\left(x_{1} y_{2}-x_{2} y_{1}\right)\left(I_{x x} I_{y y}(x, y)-I_{x y}^{2}(x, y)\right)\right]^{2} \\
& \approx {\left[\frac{1}{2} \sum_{x_{1}, x_{2}, y_{1}, y_{2}} g\left(x_{1}, y_{1}\right) g\left(x_{2}, y_{2}\right)\left(x_{1}^{2}+x_{2}^{2}\right)\right]\left[I_{x y} I_{x}(x, y)-I_{x x} I_{y}(x, y)\right]^{2} } \\
&+\left[\frac{1}{2} \sum_{x_{1}, x_{2}, y_{1}, y_{2}} g\left(x_{1}, y_{1}\right) g\left(x_{2}, y_{2}\right)\left(y_{1}^{2}+y_{2}^{2}\right)\right]\left[I_{y y} I_{x}(x, y)-I_{x y} I_{y}(x, y)\right]^{2} \\
&= \sigma^{2}\left[\left(I_{x y} I_{x}(x, y)-I_{x x} I_{y}(x, y)\right)^{2}+\left(I_{y y} I_{x}(x, y)-I_{x y} I_{y}(x, y)\right)^{2}\right],
\end{aligned}
$$




$$
C_{2}(x, y)=\left[\widehat{I_{x}^{2}}(x, y)+\widehat{I}_{y}^{2}(x, y)\right]^{2} \approx\left[I_{x}^{2}(x, y)+I_{y}^{2}(x, y)\right]^{2} .
$$

Thus the new cornerness measure becomes

$$
\Delta_{1}=\left[I_{x y} I_{x}-I_{x x} I_{y}\right]^{2}+\left[I_{y y} I_{x}-I_{x y} I_{y}\right]^{2}-K^{\prime}\left[I_{x}^{2}+I_{y}^{2}\right]^{2}, \quad \text { where } K^{\prime}=K / \sigma^{2} .
$$

Note that for most gradient operators $I_{x}$ and $I_{y}, \partial I_{x} / \partial y=0, \partial I_{y} / \partial x=0$. So that

$$
I_{x}\left(x+x_{0}, y+y_{0}\right) \approx I_{x}(x, y)+x_{0} I_{x x}(x, y), \quad I_{y}\left(x+x_{0}, y+y_{0}\right) \approx I_{y}(x, y)+y_{0} I_{y y}(x, y) .
$$

Then the cornerness measure can be simplified as follows:

$$
\begin{aligned}
C_{1}(x, y) \approx & \frac{1}{2} \sum_{x_{1}, x_{2}, y_{1}, y_{2}} g\left(x_{1}, y_{1}\right) g\left(x_{2}, y_{2}\right)\left[\left(I_{x}(x, y)-x_{1} I_{x x}(x, y),\right.\right. \\
& \left.\left.I_{y}(x, y)-y_{1} I_{y y}(x, y)\right)\left(I_{x}(x, y)-x_{2} I_{x x}(x, y), I_{y}(x, y)-y_{2} I_{y y}(x, y)\right)\right]^{2} \\
= & \frac{1}{2} \sum_{x_{1}, x_{2}, y_{1}, y_{2}} g\left(x_{1}, y_{1}\right) g\left(x_{2}, y_{2}\right)\left[\left(x_{1}-x_{2}\right) I_{x x} I_{y}(x, y)-\left(y_{1}-y_{2}\right) I_{y y} I_{x}(x, y)-\left(x_{1} y_{2}-x_{2} y_{1}\right) I_{x x} I_{y y}(x, y)\right]^{2} \\
\approx & {\left[\frac{1}{2} \sum_{x_{1}, x_{2}, y_{1}, y_{2}} g\left(x_{1}, y_{1}\right) g\left(x_{2}, y_{2}\right)\left(x_{1}^{2}+x_{2}^{2}\right)\right] I_{x x}^{2} I_{y}^{2}(x, y)+\left[\frac{1}{2} \sum_{x_{1}, x_{2}, y_{1}, y_{2}} g\left(x_{1}, y_{1}\right) g\left(x_{2}, y_{2}\right)\left(y_{1}^{2}+y_{2}^{2}\right)\right] I_{y y}^{2} I_{x}^{2}(x, y) } \\
= & \sigma^{2}\left[I_{x x}^{2} I_{y}^{2}(x, y)+I_{y y}^{2} I_{x}^{2}(x, y)\right] .
\end{aligned}
$$

Thus the new cornerness measure becomes

$$
\Delta_{2}=\left[I_{x x} I_{y}\right]^{2}+\left[I_{y y} I_{x}\right]^{2}-K^{\prime}\left[I_{x}^{2}+I_{y}^{2}\right]^{2}, \quad \text { where } K^{\prime}=K / \sigma^{2} .
$$

So that we can define the above cornerness measure as the new corner detector.

The most important improvement of the new corner detector is that it decreases the complexity greatly. Instead of calculating the three Gaussian smoothed gradient-multiple images. Only two second-order gradient-multiple images are required. The speed of the new corner detector is faster than the Plessey corner detector when the neighborhood of the second-order gradient operator is not bigger than that of the Gaussian convolution.

In the implementation we have used a parameter function $K(x, y)$ instead of the constant variable $K$ in the false corner response suppression (Wang and Brady, 1995). The parameter function $K(x, y)$ is defined as the convolution of the Gaussian operator $G(\sigma, x, y)$ with the original cornerness measure. We have known that when $K$ is a constant variable, $K$ must vary with different differentiation masks and different Gaussian convolution which means it causes the corner detector to be instable and thus not suitable to the automation process. While use usage of $K(x, y)$ increases the application scope to satisfy changing image surface and removes the man-made effect on the choice of parameter value of $K$ to balance the detection and localization performance of corner detection. The usage of $K(x, y)$ increases the detection and localization performance but slightly adds the complexity.

In all, the cornerness measure of the new corner detector is defined as follows:

$$
\Delta(x, y)=I_{x}^{2}(x, y) I_{y y}^{2}(x, y)+I_{y}^{2}(x, y) I_{x x}^{2}(x, y)-K(x, y)\left(I_{x}^{2}(x, y)+I_{y}^{2}(x, y)\right)^{2} .
$$

\section{The implementation of the new corner detector}

1. Compute the intensity gradient estimate images $I_{x}(x, y), I_{y}(x, y), I_{x x}(x, y)$ and $I_{y y}(x, y)$.

2. Compute the four gradient-multiple image: $I_{x}^{2}(x, y), I_{y}^{2}(x, y), I_{x x}^{2}(x, y)$ and $I_{y y}^{2}(x, y)$. 
3. Compute the Gaussian smoothed partial variable $K(x, y)$ :

$$
\begin{aligned}
& \Delta_{0}(x, y)=\frac{I_{x}^{2}(x, y) I_{y y}^{2}(x, y)+I_{y}^{2}(x, y) I_{x x}^{2}(x, y)}{\left(I_{x}^{2}(x, y)+I_{y}^{2}(x, y)\right)^{2}}, \\
& K(x, y)=G(\sigma, x, y) \otimes \Delta_{0}(x, y), \quad \text { where } \sigma=1.0 .
\end{aligned}
$$

4. Compute the cornerness measure

$$
\Delta=I_{x}^{2} I_{y y}^{2}+I_{y}^{2} I_{x x}^{2}-K(x, y)\left(I_{x}^{2}+I_{y}^{2}\right)^{2} .
$$

5. Output all local maximum of $\Delta(x, y)$, above a given threshold, as the locations of the detected corner points.

\section{Some properties of the new corner detector}

- The performance of gradient-direction corner detection is slightly inferior to that of the Plessey detector but the performance of localization is better than that of the Plessey detector - balance between the performance of detection and the localization.

- The corner detector is instable because of the application of constant-variable false corner response suppression.

- The corner detector algorithm needs to decide the thresholding value and the variable $K_{\text {Plessey }}$ for the false corner response suppression - one less parameter than the Plessey corner detector.

- The corner detector algorithm only requires $M^{2}\left(4 N^{2}+8\right)$ operations where the image is $M$ by $M$, the neighborhood of the gradient operators is $N^{2}$ - simpler than the Plessey corner detector.

\section{Differential geometry feature}

We have employed the differential geometry to analyze the image surface, and applied to explain the cornerness measure.

Let the image by $I(x, y), n=\nabla I /|\nabla I|$ be the edge normal and $t$ be the edge tangential, we have

$$
\frac{\partial^{2} I}{\partial n^{2}}=\frac{I_{x}^{2} I_{x x}+2 I_{x} I_{y} I_{x y}+I_{y}^{2} I_{y y}}{|\nabla I|^{2}}, \quad \frac{\partial^{2} I}{\partial t^{2}}=\frac{I_{y}^{2} I_{x x}-2 I_{x} I_{y} I_{x y}+I_{x}^{2} I_{y y}}{|\nabla I|^{2}} .
$$

So that the Kitchen's cornerness measure can be rewritten as

$$
\Delta_{\text {Kitchen }}=\frac{\partial^{2} I}{\partial t^{2}},
$$

which means that the Kitchen's cornerness measure is explicit representation for the second directional derivative in the direction orthogonal to the edge gradient.

The Wang's cornerness measure is as follows:

$$
\Delta_{\text {Wang }}=\frac{\partial^{2} I}{\partial t^{2}} /|\nabla I| \text {. }
$$

In all, the edge-related cornerness measure can be synthesized as the following form:

$$
\Delta=\frac{\partial^{2} I}{\partial t^{2}} /|\nabla I|^{n}
$$


Denote the gradient direction of $\theta(x, y)$ at any point $(x, y)$ is given by

$$
\tan (\theta)=\frac{I_{y}}{I_{x}}
$$

The partial derivatives of $\theta(x, y)$ are

$$
\theta_{x}=\frac{I_{x y} I_{x}-I_{x x} I_{y}}{I_{x}^{2}+I_{y}^{2}}, \quad \theta_{y}=\frac{I_{y y} I_{x}-I_{x y} I_{y}}{I_{x}^{2}+I_{y}^{2}} .
$$

Thus the original cornerness measure $\Delta$ of new corner detector becomes

$$
\begin{aligned}
\Delta(x, y) & =I_{x x}^{2} I_{y}^{2}+I_{y y}^{2} I_{x}^{2}-K\left[I_{x}^{2}+I_{y}^{2}\right]^{2} \\
& \approx\left[I_{x y} I_{x}-I_{x x} I_{y}\right]^{2}+\left[I_{y y} I_{x}-I_{x y} I_{y}\right]^{2}-K\left[I_{x}^{2}+I_{y}^{2}\right]^{2} \\
& =\|\nabla \theta(x, y)\|^{2}\|\nabla I(x, y)\|^{2}-K\|\nabla I(x, y)\|^{2} \\
& >0 .
\end{aligned}
$$

This inequality gives us a clue of how the gradient-direction corner detector works. In fact, the first term of the above equation measures gradient module of the image gradient direction and the gradient magnitude while the second term of equation provides a measure of the gradient magnitude. The difference of these two terms cancels the false corner response, and leave a clean corner response. Hence the aim of the corner detection is reduced to look for the maximum whenever the above inequality is found.

We exclude the constraint of false corner response suppression, the cornerness measure of new corner detector can be expressed as follows:

$$
\Delta(x, y)=\frac{I_{x y}^{2} I_{x}^{2}+I_{y y}^{2} I_{x}^{2}}{\left[I_{x}^{2}+I_{y}^{2}\right]^{2}} \approx \frac{\left[I_{x y} I_{x}-I_{x x} I_{y}\right]^{2}+\left[I_{y y} I_{x}-I_{x y} I_{y}\right]^{2}}{\left[I_{x}^{2}+I_{y}^{2}\right]^{2}}=\|\nabla \theta(x, y)\|^{2},
$$

which means that the cornerness measure of new corner detector is simplify the gradient module of the image gradient direction. Thus we call it the gradient-direction corner detection.

So that the gradient-direction cornerness is simply that: based on the measure of the gradient module of the image gradient direction, make the constraint of the false corner response suppression to make a clear corner response.

\section{Experiments}

In this section, we give some experimental results obtained by running some corner detectors on the corner models, synthetic and real images.

Table 1

Average number of detected corners for 100 noisy $90^{\circ}$ corner models (the table show that the gradient-direction detector has fair detection performance, stable in most conditions (above $85 \%$ ))

\begin{tabular}{llllll}
\hline White noise $(\%)$ & Kitchen & Beaudet & Deriche & Plessey & Gradient-direction \\
\hline$\sigma=0$ & 1 & 1 & 1 & 1 & 1 \\
$\sigma=2$ & 1 & 1 & 1 & 1 & 1 \\
$\sigma=5$ & 1 & 1.02 & 1 & 1 & 0.92 \\
$\sigma=10$ & 1 & 1.99 & 1 & 1 & 0.87 \\
$\sigma=20$ & 1 & 4.20 & 1.19 & 1 & 0.89 \\
\hline
\end{tabular}


Table 2

Average number of detected corners for 100 noisy $45^{\circ}$ corner models (the table show that the gradient-direction detector has fair detection performance, comparable with the Plessey detector)

\begin{tabular}{llllll}
\hline White noise (\%) & Kitchen & Beaudet & Deriche & Plessey & Gradient-direction \\
\hline$\sigma=0$ & 1 & 1 & 1 & 1 & 1 \\
$\sigma=2$ & 1 & 1 & 1 & 1 & 1 \\
$\sigma=5$ & 1 & 1.05 & 1.13 & 1 & 1 \\
$\sigma=10$ & 1 & 3.07 & 1.10 & 1 & 1 \\
$\sigma=20$ & 1 & 6.30 & 1.02 & 1 & 1.04 \\
\hline
\end{tabular}

\subsection{Results on corner models}

In order to compare the performance of detection, we have performed a statistical analysis on the corner detection. We have considered 100 noisy $90^{\circ}$ and $45^{\circ}$ corner models corresponding to five different white noise levels $(0 \%, 2 \%, 5 \%, 10 \%$ and $20 \%)$ to find out the average detected corner points. From Tables 1 and 2 we can make the conclusion that the new corner detector has a fair detection performance, stable in the most conditions (above 85\%).

In order to compare the performance of localization, we have performed the Gaussian convolution with the original images before the corner detection to five different Gaussian scales $(0,1,2,5$, 10). From Tables 3 and 4 we can make the conclusion that the new corner detector has a better localization performance than the Plessey detector, and more stable.

Table 3

Corner coordinate for the Gaussian scaled $90^{\circ}$ corner model (the table show that the gradient-direction detector has better localization performance than the Plessey detector)

\begin{tabular}{llllll}
\hline Gaussian scale & Kitchen & Beaudet & Deriche & Plessey & Gradient-direction \\
\hline$\sigma=0$ & $(0.635,0.635)$ & $(0.907,0.907)$ & $(0,0)$ & $(0.882,0.882)$ & $(0.892,0.892)$ \\
$\sigma=1$ & $(0.736,0.736)$ & $(1.219,1.219)$ & $(0,0)$ & $(0.961,0.961)$ & $(0.966,0.966)$ \\
$\sigma=2$ & $(1.234,1.234)$ & $(2.146,2.146)$ & $(0,0)$ & $(2.011,2.011)$ & $(1.883,1.883)$ \\
$\sigma=5$ & $(3.749,3.749)$ & $(5.482,5.458)$ & $(0,0)$ & $(12.132,12.132)$ & $(4.858,4.858)$ \\
$\sigma=10$ & $(7.912,7.912)$ & $(11.275,11.276)$ & $(0,0)$ & $(28.401,28.401)$ & $(9.652,9.653)$ \\
\hline
\end{tabular}

Table 4

Corner coordinate for the Gaussian scaled $45^{\circ}$ corner model (the table show that the gradient-direction detector has more stable localization performance than the Plessey detector)

\begin{tabular}{|c|c|c|c|c|c|}
\hline Gaussian scale & Kitchen & Beaudet & Deriche & Plessey & Gradient-direction \\
\hline$\sigma=0$ & $(0.814,2.057)$ & $(0.851,3.123)$ & $(0,1)$ & $(0.892,1.854)$ & $(1.000,1.862)$ \\
\hline$\sigma=1$ & $\begin{array}{l}(0.926,2.511) \\
(1.918,4.861)\end{array}$ & $(0.977,3.350)$ & $(0,1)$ & $(0.895,2.269)$ & $(1.000,1.930)$ \\
\hline$\sigma=2$ & $\begin{array}{l}(1.178,3.149) \\
(1.906,4.793)\end{array}$ & $(1.879,5.157)$ & $(0,1)$ & $(1.389,3.538)$ & $(1.991,3.962)$ \\
\hline$\sigma=5$ & $\begin{array}{l}(4.150,10.305) \\
(4.802,11.792) \\
(6.707,16.290)\end{array}$ & $(4.965,12.387)$ & $(0,1)$ & $\begin{array}{l}(4.487,11.067) \\
(4.601,11.303)\end{array}$ & $\begin{array}{l}(4.185,8.787) \\
(4.677,10.101)\end{array}$ \\
\hline$\sigma=10$ & $\begin{array}{l}(9.238,22.572) \\
(9.641,23.482)\end{array}$ & $(10.234,25.025)$ & $(0,1)$ & $\begin{array}{l}(15.041,34.357) \\
(15.933,36.296)\end{array}$ & $\begin{array}{l}(9.091,19.625) \\
(9.499,20.693)\end{array}$ \\
\hline
\end{tabular}




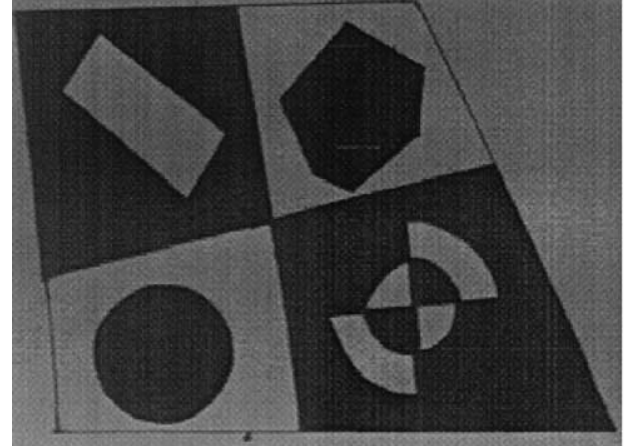

(a) The Original Noisy Image

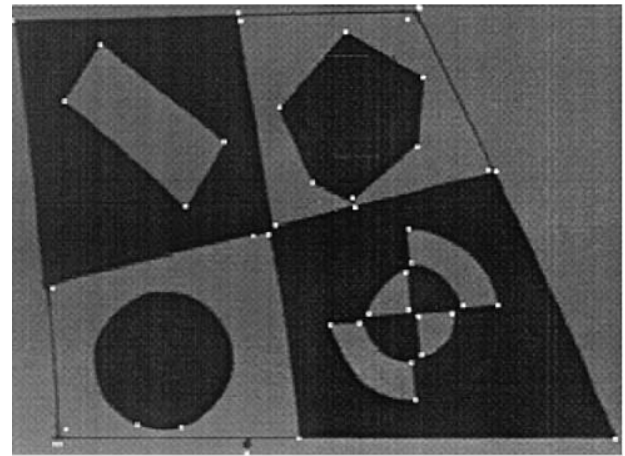

(c) The Beaudet Operator

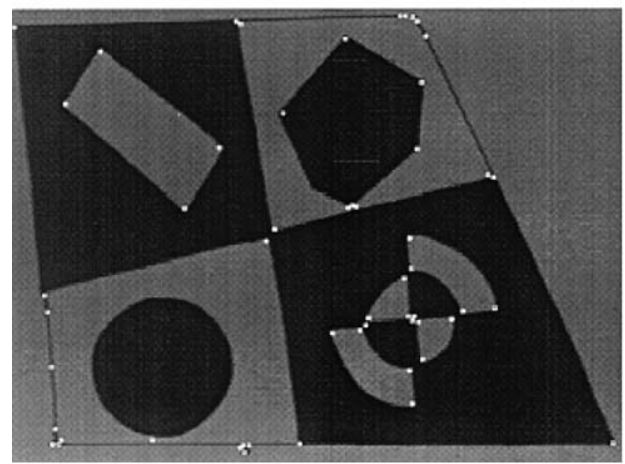

(e) The Plessey Operator

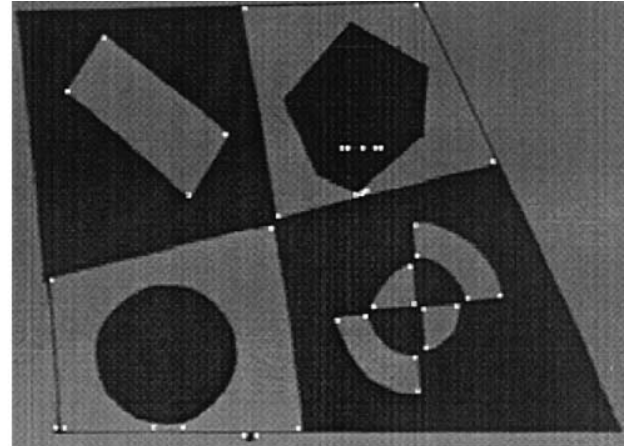

(b) The Kitchen and Rosenfeld Operator

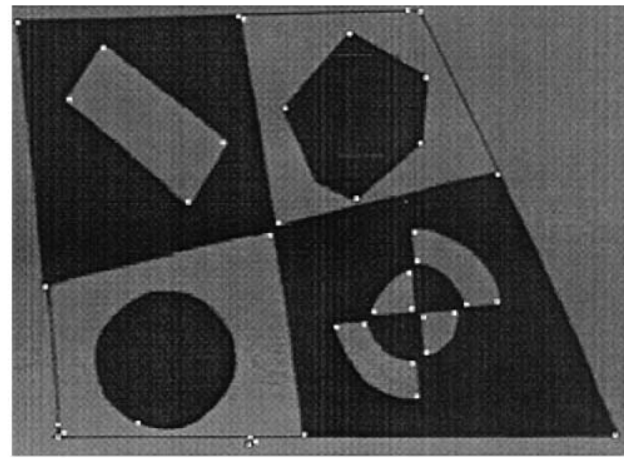

(d) The Deriche Operator

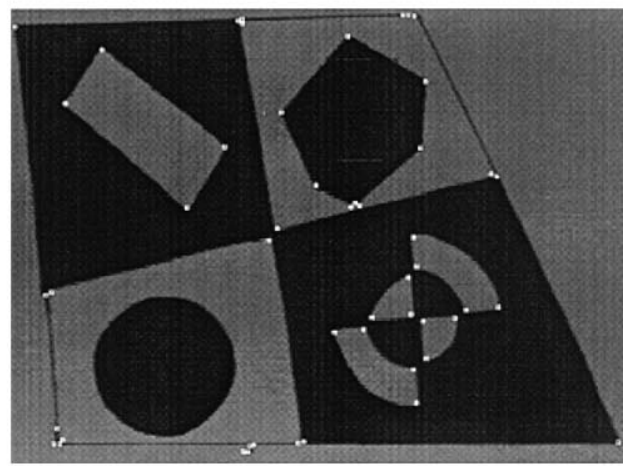

(f) The Gradient-Direction Operator

Fig. 1. The results of corner operator to noisy synthetic image. (Kitchen detector falsely detects points in the noisy line and fails to detect vertexes in the polygon. Beaudet detector fails to detect one vertex at the right bottom. So does Deriche detector. Plessey detector falsely detects noisy edge points and fails to detect one vertex in the polygon. Only the gradient-direction detector has well detected and positioned all the features.) 


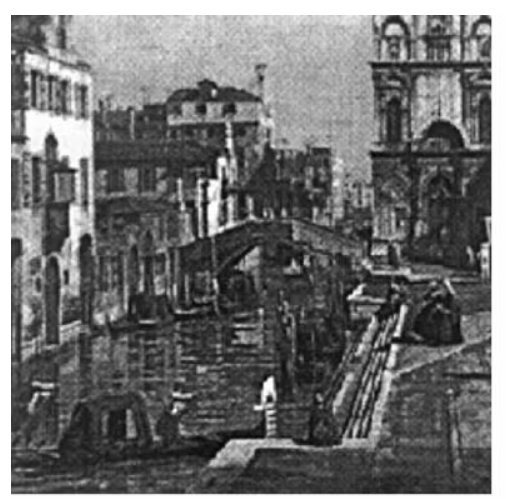

(a) The Original Outdoor Scene

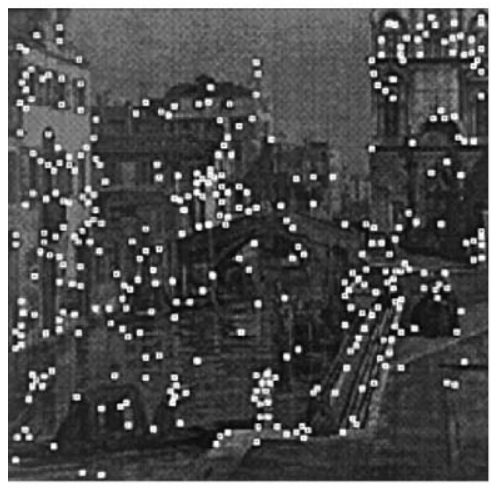

(c) The Beaudet Operator

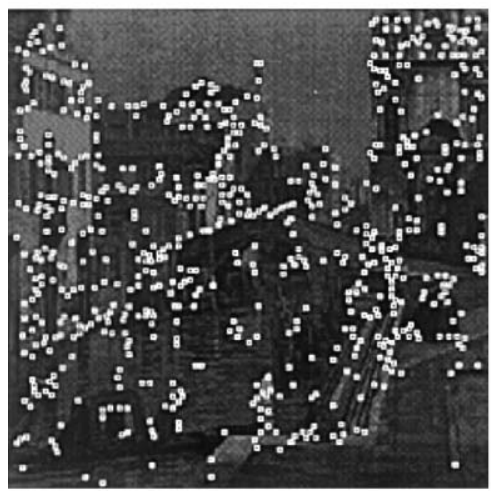

(e) The Plessey Operator

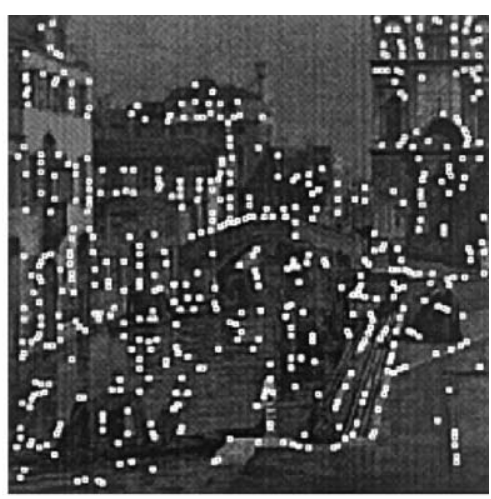

(b) The Kitchen and Rosenfeld Operator

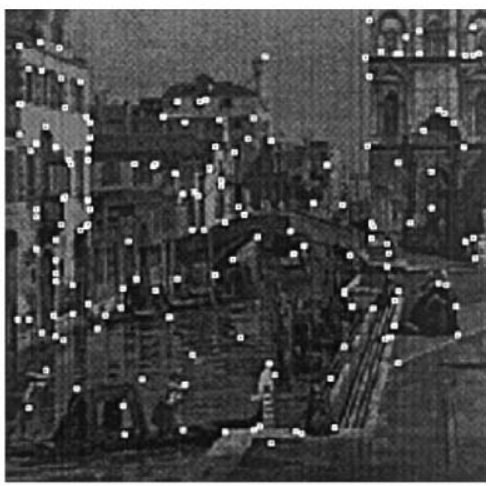

(d) The Deriche Operator

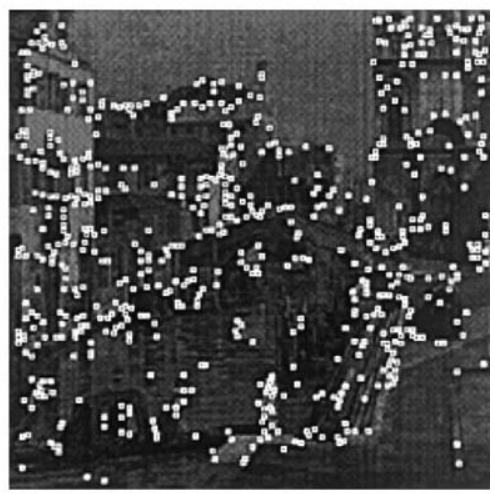

(f) The Gradient-Direction Operator

Fig. 2. The results of corner operator to outdoor scene. (Kitchen detector fails to detect some weak corners. Beaudet detector falsely response to the noise too much. The performance of Plessey detector and the gradient-direction detector are better than the others. The gradient-direction detector detects even more subtle corner points with accurate locations than Plessey detector.) 


\subsection{Results on synthetic and real images}

To illustrate the efficiency of the gradient-direction corner detector on the synthetic image, Fig. 1 shows an example of the promising results we have obtained by running some corner detectors on a synthetic image. The results show that the new corner detector keeps the best balance between the performance of detection and localization.

To illustrate the efficiency of the gradient-direction detector on the real image, Fig. 2 shows an example of the promising results we have obtained by running some corner detectors on the real images. The results of the gradient-direction detector are very stable and suggest that the matching process will be much easier and more reliable using these feature.

\section{Discussion}

We have proposed a new approach called gradient-direction corner detector. Based on the measure of the gradient module of the image gradient direction, it simply imposes the constraint of the partial variable false corner response suppression to obtain a clear corner response. The proposed approach has been tested on both synthetic and real images with compare to the other corner detectors. The performance of some corner detectors are summarized here

- Kitchen corner detector. The performance is not desirable. The experiments show that it cannot distinguish the corner points from the noisy edge points, which means it cannot meet the requirement of corner detection.

- Beaudet corner detector. The Beaudet corner detector is more sensitive to the noise effect than the others. However it can correctly detect the corner points even in the case of large Gaussian convolution, more stronger than the others. It is quite stable because it provides local maximums in all directions. And more important, it is the simplest corner detector of all. But unfortunately, the experiments show that the performance is not as good as expected, mainly because of the complexity of the corner structure in the real image.

- Plessey corner detector. The Plessey corner detector has the best performance of detection, but the performance of localization is not good enough, particularly in the case of large Gaussian convolution. Even more the application of constant-variable false corner response suppression causes it to be unstable.

- Gradient-direction corner detector. The gradient-direction corner detector is comparable with the Plessey corner detector with respect to the performances of detection but it has better performance of localization, particularly in the case of large Gaussian convolution. Even more it require fewer intensive Gaussian convolution than the Plessey corner detector, hence reduces the complexity. Moreover, it imposes the constraints of partial-variable false corner response suppression, hence improves the stability.

We conclude that the gradient-direction corner detector is most suitable for the corner detection, mostly with application in real-time image processing.

\section{References}

Alison Noble, J., 1988. Finding corners. Image and Vision Computing 6 (2), 121-128.

Alison Noble, J., 1989. Description of image surfaces. Ph.D. Thesis Oxford University.

Beaudet, P.R., 1978. Rational invariant image operators. In: Fourth International Conference on Pattern Recognition, pp. 579-583.

Cooper, J., Svetha, Kitchen, L., 1993. Early jump-out corner detectors. IEEE Transactions on PAMI 15, 823-828.

Deriche, R., Giraudon, G., 1993. A computational approach for corner and vertex detection. International Journal of Computer Vision 10 (2), 101-124. 
Guiducci, A., 1988. Corner characterization by differential geometry techniques. Pattern Recognition Letters 8, 311-318.

Hsin-Teng, Hu, W.-C., 1996. A rotationally invariant two-phase scheme for corner detection. Pattern Recognition 28, 819-828.

Kitchen, L., Rosenfeld, A., 1982. Gray-level corner detection. Pattern Recognition Letters 1, 95-102.

Lin, R.-S., Chu, C.-H., Hsueh, Y.-C., 1998. A modified morphological corner detector. Pattern Recognition Letters 19, $279-286$.

Moravec, H.P., 1977. Towards automatic visual obstacle avoidance. In: Fifth International Joint Conference On Artificial Intelligent, p. 584.

Ponce, J., Brady, M., 1985. Towards a surface primal sketch. In: IEEE International Conference on Robotics and Automation, pp. $420-425$.

Rattarangsi, A., Chin, R.T., 1992. Scales-based detection of corners of planar curves. IEEE Transactions on PAMI 14 (4), $430-449$.

Rohr, K., 1992. Modeling and identification of characteristic intensity variations. Image and Vision Computing.

Singh, A., Shneier, M., 1990. Gray level corner detection a generalization and a robust real time implementation. Computer Vision, Graphics, and Image Processing 51, 54-69.

Wang, H., Brady, M., 1995. Real-time corner detection algorithm for motion estimation. Image and Vision Computing 13 (9). 